\title{
Design and Simulation of Optical Current Transformer Based on Mixed Programming of Ansoft and VB
}

\author{
Linyang Chen, Qifeng Xu \\ Electric Engineering and Automation College, Fuzhou University, Fuzhou, China \\ Lychen1992@126.com
}

\begin{abstract}
In order to optimize the design of optical current transformer and cut down the development cycle, the electromagnetic design software with Ansoft and VB is developed in this paper. The interface technology between Ansoft and VB is proposed to achieve the mixed programming, obtain the visualization interface of input data, simplify the electromagnetic design and make the output date clear and easy to read.
\end{abstract}

Keywords-optical current transformer; ansoft maxwell; VB; electromagnetic design

\section{INTRODUCTION}

Optical Current Transformer (OCT) has the advantages of good insulation, wide frequency band and no magnetic saturation, so it will become the ideal substitution of conventional CT which used in the high voltage and ultrahigh voltage power systems.

In the published literatures of the OCT, the research works are mainly aimed at the optimization design of the structure of sensing head and the analysis of the internal magnetic field [1, 2, 3]. In the experimental process of optimizing the design of the OCT, the model design and electromagnetic calculation with multi-variables and multisteps, in repeated operations and the other characteristics are very complicated. It makes the design work of the OCT very hard. Therefore, the design software of the OCT must include a powerful simulation, which is the main basis for performance optimization and structural design.

At present, the main auxiliary design and analysis software of the OCT are: Ansoft Maxwell, ANSYS, Matlab and etc. Because of the operation of Ansoft Maxwell is quite complicated in modeling, assigning the excitation source and adding the boundary conditions, it is just a waste of time to do such a series of repeated design. Therefore, it is required to find a simple way to improve efficiency.

In order to solve the problems of complicated modeling and applying inconveniently with Ansoft Maxwell, the software of optical current transformer's electromagnetic field analysis is developed which is completely based on Windows environment in a method of object oriented programming. The main menu of the software is compiled with VB language. The software quotes the magnetic field calculation file through the command of shell.

\section{Method ON Programming BASEd ON ANSOFT MAXWELL AND VB}

\section{A. The Script Function of Ansoft Maxwell}

Ansoft Maxwell provides a script function which is equal to the DOS batch processing command. The script file is plain text file which doesn't provide the visualization function so it takes up much more time to compile. The language used by the script file is Visual Basic Script (VB script) which is the scripting language of VB. This scripting language is not a completely independent programming one, which only include a series of the basic functions of programming language. But the biggest advantage of the scripting language is that the code is all set up by the pure text so it can be very easy to edit and modify. The text file can be quoted by VB, so the seamless link between Ansoft Maxwell and VB can be achieved.

\section{B. Axtive X Technology}

In the environment of VB, Ansoft Maxwell can be quoted as a ActiveX component of VB through the ActiveX automation interface. At this point, Ansoft Maxwell can be used as the automation server and be controlled by VB [4]. The software interface language between Ansoft Maxwell and VB can be achieved through the following code.

Set oAnsoftApp = CreateObject

(“AnsoftMaxwell.MaxwellScriptInterface”)

SetoDesktop = oAnsoftApp.GetAppDesktop()

Visual Basic can directly enter the Ansoft Maxwell through executing the above code. It is easy to modify and set up. In addition, it is also able to observe and modify the simulation in real time.

\section{ElECTROMAGNETIC DeSIGN SOFTWARE OF OptICAL CURRENT TRANSFORMER}

\section{A. Flow Diagram of Software Design}

Visual Basic(VB) is a kind of commonly used programming development tool which can make the originally difficult work of programming and software development to be easy. The initial programming work is to rely on a full set of code which has a large amount of engineering. Through the VB's visual design platform it turns the past pure code programming into a visual programming. It packages some functions in the form of controls. The designer only needs to draw a variety of objects on the screen according to design requirements [5]. 
VB is characterized by good interaction and the introduction of object-oriented and event driven programming ideas, so the designer who is not specifically engaged in the development of the program is also easy to manage it. The shortcomings of $\mathrm{VB}$ are the tedious programming language and the weak computing power. In particular, there is no special graphics processing module which brings a lot of inconveniences to engineering applications. However, the graphics processing and analyses of the magnetic field profiles are absolutely required in the simulation analysis of the OCT so it is combined with a powerful graphics processing of Ansoft Maxwell and a powerful computing ability with the good human-computer interaction interface of VB to realize the module and visualization which can give a full play to the advantages of these two kinds of software. And it can optimize the structure of the software and improve work efficiency. The design flow diagram is shown in Figure. 1.

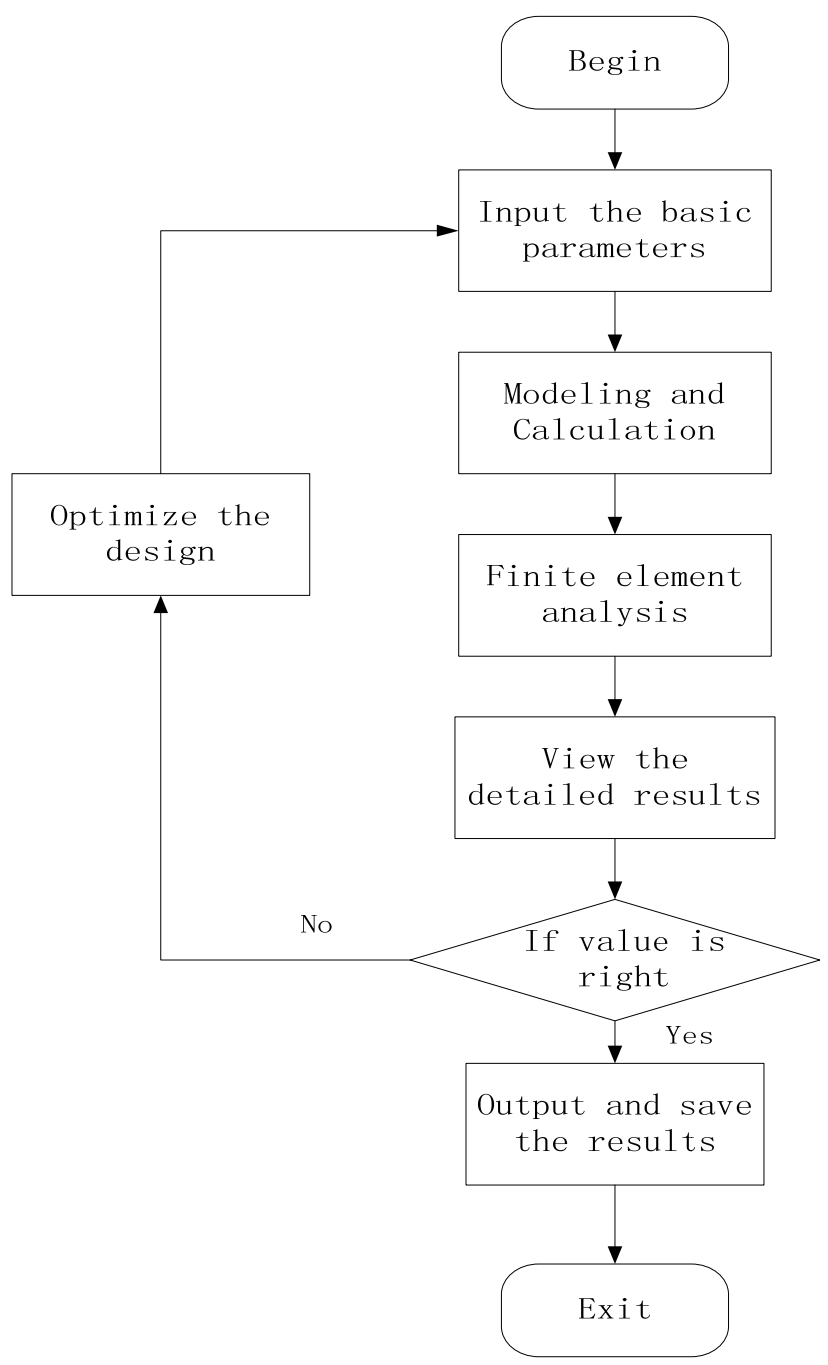

Figure 1. Steps for designing

\section{B. Software Interface Design}

A project including a main form and multiple auxiliary forms is created in the VB environment. The title bar of the main form has two menu buttons which are used to hold the input parameters and open the file with the original simulation experiment data. Besides, the main form has four main function buttons which are named as modeling calculation, finite element analysis, detailed calculation results and optimization design respectively. Figure. 2 shows the interface of the software. The function of modeling calculation will model as the parameters inputted by the user. The function of finite element analysis can get all kinds of performance curves and data tables. What' more, Engineer can optimize the structure of the sensing head after analyzing the detailed results [6].

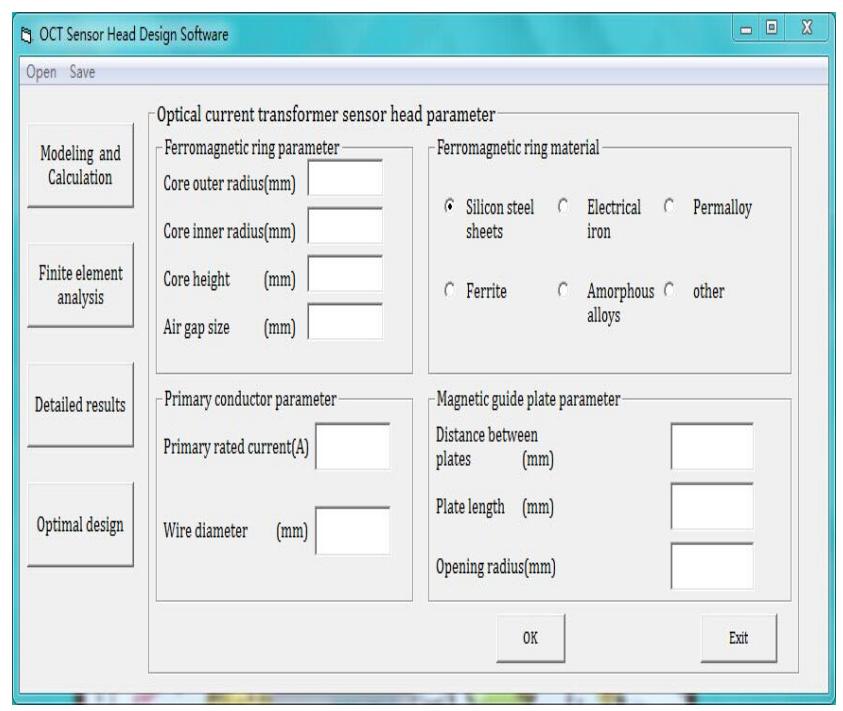

Figure 2. Form interface

\section{Software Programming}

1) Data transmission between Ansoft Maxwell and VB.

The data transmission between Ansoft Maxwell and VB is realized through reading and writing the text file. The text file is the script file which is generated by the Ansoft Maxwell mentioned above. The idea of software design is recording the Ansoft Maxwell script, that needs to be called at the beginning of the programming according to the characteristics of the magnetic field analysis of the optical current transformer sensing head. And the design parameters that are involved in the script file are set as variables. Because the Ansoft Maxwell script can be opened by the notepad, VB can search for the corresponding variable name and assign the relevant input parameter values to the preset variable. The specific parameters have the outer radius of core, the inner radius of the core, the height of the core, the size of the air gap and so on.

When the user clicks on the ok button, the software begins to read and write the text file between Ansoft Maxwell and VB. Code is as follows.

Open “Original model 1” For Input As \#1 
Do while Not EOF(1)

Line Input \#1, line

If InStr(line,” Core outer radius”) Then

Line =Replace(line,” Core outer radius”,Text1.Text)

End If

Close \#1

Open "custom model 1" For Output As \#1

Print \#1, s;

Close \#1

By executing the above statement, VB can assign the various parameters inputted originally by the user to the corresponding variable. And then Ansoft Maxwell will be directly opened to model and analyze, user can also observe and modify the simulation in real-time. The Called code is as follows.

Shell" wscript "Script path"”

2) Finite element analysis and result output mode.

The design of the sensing head of optical current transformer needs to obtain the visualized result of the graphic and the list so the analysis results of the sensing head are mainly presented in the form of curves and Excel lists. When the user needs to carry out the finite element analysis, the click of the button will pop up the auxiliary form for the analysis of the selection of the type as show in Figure.3 [7].

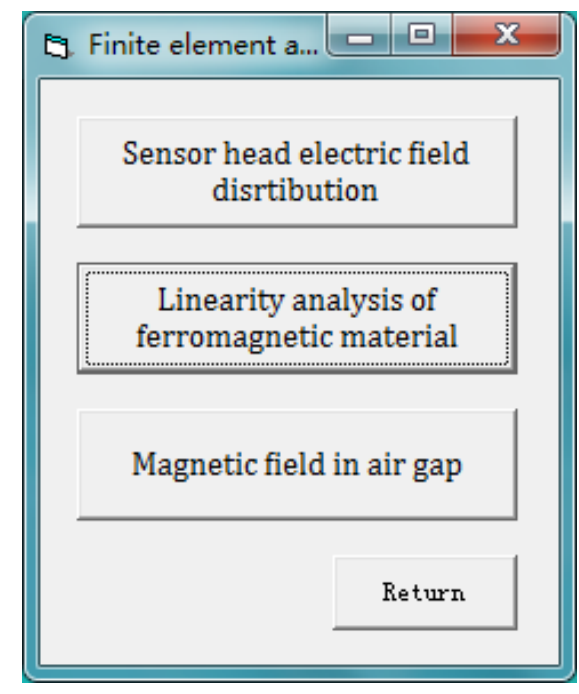

Figure 3. Finite element analysis interface

Because it is needed to analyze the distribution curve of the magnetic field and needed to know its specific value, therefore the performance of the results can be selected according to the needs of users. Figure. 4 shows the magnetic field intensity distribution at the center of the air gap. The distribution curve is obtained by Ansoft Maxwell and presented at the interface of the software by VB.

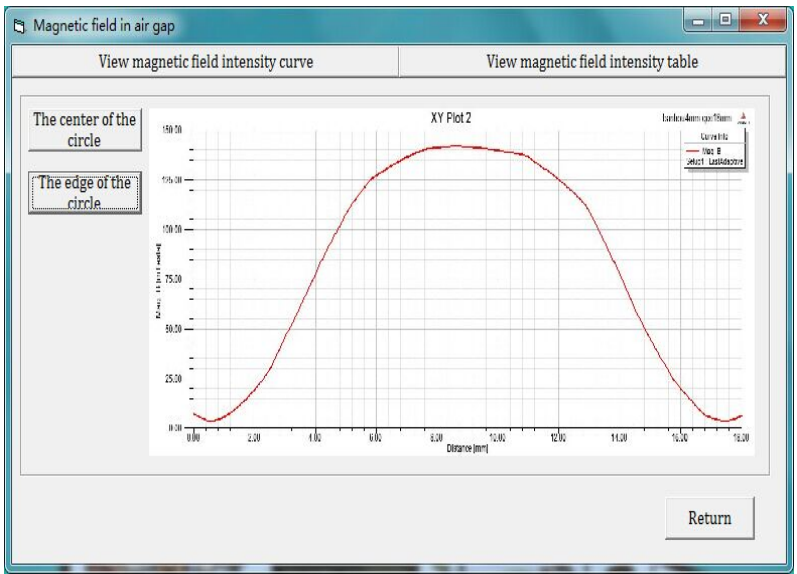

Figure 4. Magnetic field distribution at the center of the air gap

In addition, the magnetic field intensity valued of the sample points are presented in the form of a list. Taking into account the wide range of Excel applications and powerful data processing capabilities, Excel is used to storage data. The method is to store the simulation data in the form of Excel by Ansoft Maxwell, and then VB calls the Excel table for the result output. The code is as follows.

Dim xlsApp As Excel.Application

Dim xlsWorkbook As Excel.Workbook

Dim xlssheet As Excel.Worksheet

Dim AppExcel As Object

Set xlsApp = CreateObject("Excel.Application")

Set xlsWorkbook

xlsApp.Workbooks.Open(“D: |Result.xls”)

Set xlsBook $=$ xlExcel. Worksheets $(1)$

Text1.Text $=$ xlSheet.Cells $(1,1)$. Value

Text1.Text = Text1.Text \& “ADD”

xlSheet. Cell(1,1).Value = Text1.Text

Application.DisplayAlerts $=$ False

xlsApp. Visible $=$ True

xlsApp.Range(“A1”).Select

\section{APPLICATION EXAMPLES}

Using the software developed in this paper, we designed and optimized the collection magnetism type optical current sensing head. Examples are as follows.

According to the different shape of the hysteresis loop (the difference between the residual magnetism and the coercive force), ferromagnetic materials can be divided into soft magnetic materials and hard magnetic materials. The high magnetic permeability materials of silicon steel sheets, Amorphous alloy and perm alloy are commonly used in the ferromagnetic ring. Through the comparison of the simulation results of the software, the ferromagnetic material is employed which has the best linearity. The model dimensions of ferromagnetic ring: core outer radius $70 \mathrm{~mm}$, core inner radius $40 \mathrm{~mm}$, core height $30 \mathrm{~mm}$ and gap size $35 \mathrm{~mm}$. The magnetic field simulation is carried out on the sensing head of the three materials, respectively. The results are shown in Figure. 5, Figure. 6 and Figure. 7. 


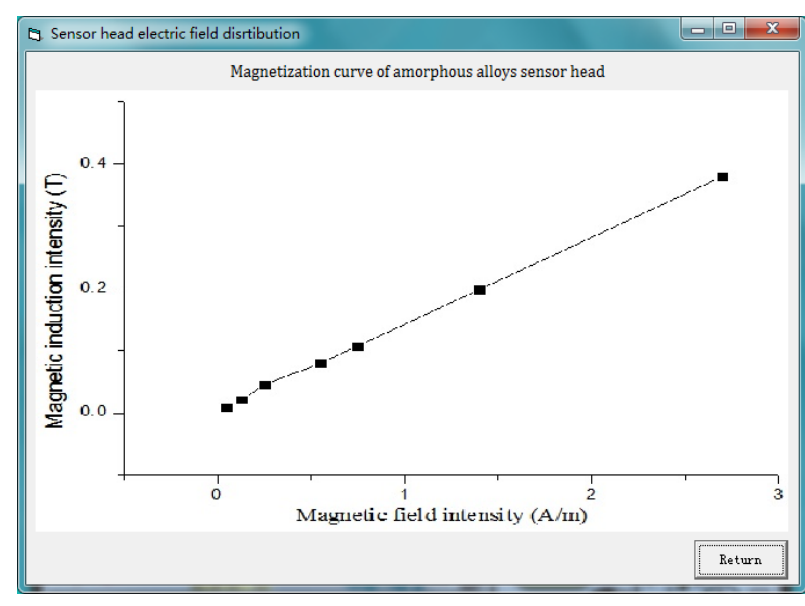

Figure 5. Magnetization curve of amorphous alloys sensor head

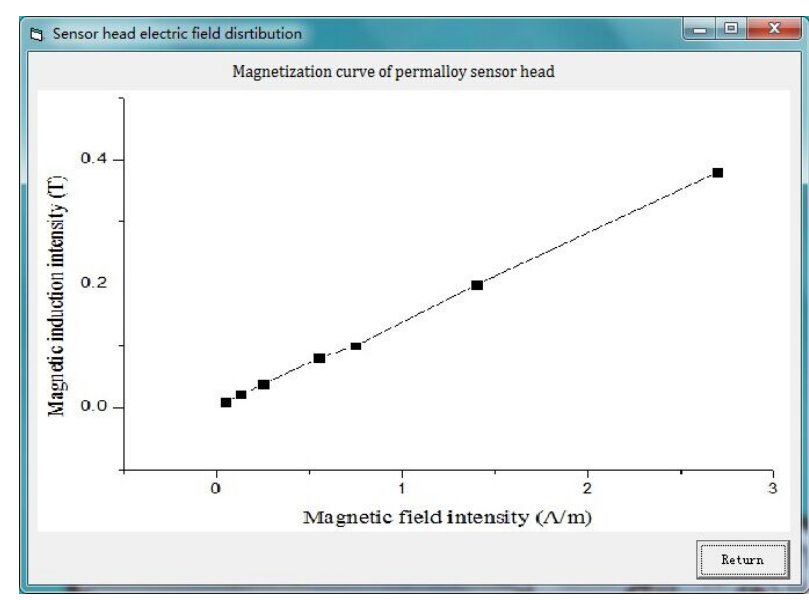

Figure 6. Magnetization curve of permalloy sensor head

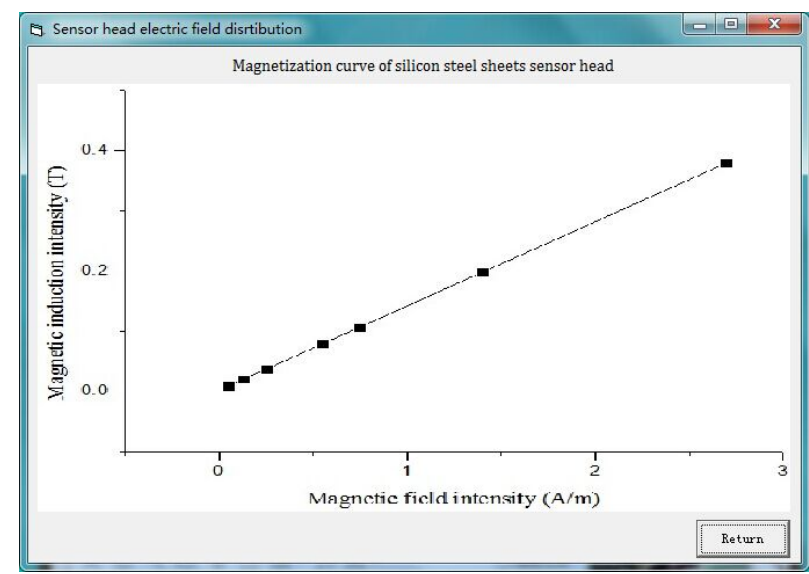

Figure 7. Magnetization curve of silicon steel sheets sensor head

From the figures, we can know that the silicon steel sheets sensing head has the best linearity. However, the degree of linearity about the amorphous alloy and perm alloy are bad in the small magnetic field or in the middle section of the magnetic field. In conclusion, we should choose the silicon steel sheets or amorphous alloy considering the degree of linearity.

In addition, the length of the air gap directly affects the intensity of magnetic field, and is closely related to the measurement accuracy of the OCT. Therefore, we used the software to optimize the design of the air gap. The opening dimensions are $20 \mathrm{~mm}, 25 \mathrm{~mm}, 30 \mathrm{~mm}, 35 \mathrm{~mm}, 40 \mathrm{~mm}$ and $45 \mathrm{~mm}$. The results are shown in Table I.

From the simulation data of Table I, it can see that the magnetic induction intensity in the air gap will decrease with the increase of the air gap size. The magnetic field distribution of the air gap varies with the length of the air gap too. When the air gap is too small or too large, the magnetic field distribution is not uniform, and it will have a negative impact on the measurement of the OCT. Considering the magnetic field uniformity and the magnitude of the magnetic field, it is concluded that the air gap length is set within the range of $25 \mathrm{~mm} \sim 35 \mathrm{~mm}$. It can guarantee the uniformity of the magnetic field distribution under the precondition of satisfying the magnetic induction intensity.

TABLE I. COMPARISON OF THE MAGNETIC INDUCTION INTENSITY OF THE FERROMAGNETIC RING WITH THE DIFFERENT AIR GAP SIZE

\begin{tabular}{|l|c|c|}
\hline Gas length(mm) & $\begin{array}{c}\text { Magnetic field } \\
\text { distribution }\end{array}$ & $\begin{array}{c}\text { Magnetic induction } \\
\text { intensity of air gap }(\mathbf{m T})\end{array}$ \\
\hline 20 & Average & 59.443 \\
\hline 25 & More uniform & 47.057 \\
\hline 30 & Uniform & 39.921 \\
\hline 35 & Uniform & 32.160 \\
\hline 40 & Average & 28.393 \\
\hline 45 & Average & 23.310 \\
\hline
\end{tabular}

Through a series of optimization design of the OCT, we established the simulation model of ferromagnetic ring and analyzed the degree of linearity. The model parameters of ferromagnetic ring has a core outer radius $70 \mathrm{~mm}$, core inner radius $40 \mathrm{~mm}$, core height $30 \mathrm{~mm}$, gap size $30 \mathrm{~mm}$, magnetic guide plate thickness $5 \mathrm{~mm}$, hole radius $2.5 \mathrm{~mm}$, primary rated current 1000A,wire diameter 40mm and silicon steel sheets. At the $1 \%, 5 \%, 10 \%, 20 \%, 100 \%, 120 \%$ primary rated current the magnetic field intensity is fixed at the center of the path of the light. The magnetic field intensities are as follows.

TABLE II. SimUlation RESUlTS OF DIFFERENT CURRENT INTENSITY

\begin{tabular}{|l|c|c|c|}
\hline $\begin{array}{c}\text { Primary } \\
\text { rated } \\
\text { current(A) }\end{array}$ & $\begin{array}{c}\text { Magnetic field } \\
\text { intensity(A/m) }\end{array}$ & $\begin{array}{c}\text { Ideal magnetic } \\
\text { field intensity(A/m) }\end{array}$ & $\begin{array}{c}\text { Relative } \\
\text { error(\%) }\end{array}$ \\
\hline 10 & 407.9 & 408 & 0.0245 \\
\hline 50 & 2241.2 & 2242 & 0.0357 \\
\hline 100 & 4534.4 & 4536 & 0.0353 \\
\hline 200 & 9102.6 & 9106 & 0.0373 \\
\hline 1000 & 46273.2 & 46292 & 0.0406 \\
\hline
\end{tabular}




\begin{tabular}{|c|c|c|c|}
\hline $\begin{array}{c}\text { Primary } \\
\text { rated } \\
\text { current(A) }\end{array}$ & $\begin{array}{c}\text { Magnetic field } \\
\text { intensity(A/m) }\end{array}$ & $\begin{array}{c}\text { Ideal magnetic } \\
\text { field intensity(A/m) }\end{array}$ & $\begin{array}{c}\text { Relative } \\
\text { error(\%) }\end{array}$ \\
\hline 1200 & 54934.1 & 54959 & 0.0453 \\
\hline
\end{tabular}

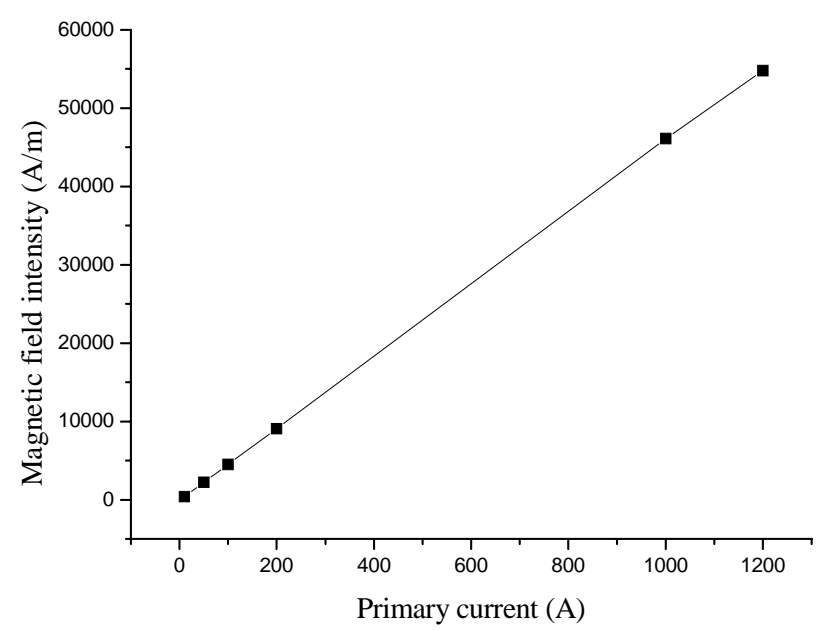

Figure 8. Response of primary rated current and air-gap magnetic field intensity

From the experimental data the response of this structure to primary current has a $0.1 \%$ of linearity.

\section{CONCLUSION}

In this paper it is fully combined the powerful finite element simulation function with the simple visual operation interface by using the interface technology of Ansoft Maxwell and VB according to the design principle and method of the optical current transformer sensing head. It is a new approach to solve the contradiction between the general program and the special needs. The method has the advantages of high efficiency, high speed, powerful image processing function and so on. The most important is to cut down the time of learning how to operate the software of Ansoft Maxwell proficiently. It shortens the development cycle of the product and reduces the probability of error in the process of model design. The feasibility of the software is verified by application examples of the collection magnetism type optics current sensing head, and it can meet the accuracy of $0.1 \%$.

\section{REFERENCES}

[1] J. Y. Wang, Z. Z. Guo, and H. B. Li, "Structure optimization of optical current transformer with magnetic concentrator ring," Electric Power Automation Equipment. Nanjing, vol. 31, pp. 23-26, Sept 2011.

[2] H. L. Guan, Q. F Shang, and Y. H. Yang, "Ferromagnetic ring concentrator sensor head of hybrid optical current transformer," Electric Power Automation Equipment. Nanjin, vol.25, pp. 30-32, Feb 2005.

[3] H. Katsukawa, H. Ishikawa, H. Okajima, T. W. Cease, "Development of an optical current transducer with a bulk type faraday sensor for metering," IEEE Transactions on Power Delivery, vol.11, pp. 702707, April 1996.

[4] Y. P. Liang and C. Liu, "Electromagnetic design method for permanent magnet synchronous motor based on Matlab and VB mixed programming," Journal of Harbin University of Science and Technology. Harbin, vol.15, pp. 25-29, Dec 2010.

[5] R. H. Gao, Y. Zhang and H. Luo, "Tower crane boom's parametric design based on VB and ANSYS,” Mechanical Engineering and Automation. Taiyuan, pp. 12-13, Feb 2008.

[6] H. Guo and Q. Xue, "Development of optimum calculation software of current transformers”, Transformer. Shenyang, vol. 40, pp. 18-22, June 2003.

[7] C. Li and Q.F Xu, "Electromagnetic-Thermal coupled analysi of optical current transducer with magnetic concentrator”, Power System Technology. Peking, vol. 38, pp. 2858-2866, Oct 2014. 